\title{
Massive Binaries
}

\author{
Anthony F. J. Moffat \\ Département de physique, Université de Montréal, \\ C.P. 6128, Succursale Centre-Ville, Montréal, QC, H3C 3J7, Canada
}

\begin{abstract}
As with all binaries, those that contain massive stars reveal various degrees of interaction, depending mainly on orbital separation and age, although things happen much faster in massive binaries. Those massive binaries with initial periods exceeding $\sim 10$ years generally only interact via wind-wind collisions, with little or no effect on their subsequent evolution (unless located in dense clusters). Shorter-period systems show even stronger wind-wind collisions as a rule, but also interact more directly via Roche Lobe Overflow or Common Envelope, with dramatic effects on their evolution. If we didn't have binaries among massive stars, we would be missing a whole host of interesting phenomena in the Universe, such as sources of enhanced stellar X-ray or non-thermal radio emission, WR dust-spirals, inverse mass-ratios, very rapid spin, rejuvenation and massive blue-stragglers, enhanced cluster dynamics, many runaways and possibly even SMBHs and GRBs! On the other hand, non(or little)-interacting massive binaries are also useful to provide information on Star-Formation processes and determination of stellar parameters (such as the mass) that would otherwise be difficult or impossible to obtain from single stars. In this review, I highlight some of the developments that have occurred during the past few years since the last IAU Symposium on Massive Stars in 2002.
\end{abstract}

Keywords. binaries: general - stars: early-type

\section{Introduction}

\subsection{Defining "massive" in binaries}

Most researchers at this meeting will agree that "massive" means having an initial mass on the Zero-Age Main Sequence (ZAMS) that leads a star to implode/explode as a corecollapse or pair-instability supernova. In the case of single stars, this means $M_{i}>8 M_{\odot}$ (although this limit may be slightly higher, depending on which stars become white dwarfs (WDs) instead: note the possible impact of the recent discovery of pure-carbon WDs: Dufour et al. 2007). Furthermore, in the case of close binaries, the primary could prematurely lose mass via Roche-lobe overflow (RLOF) or common envelope (CE) processes, which could increase the required limit slightly (Vanbeveren et al. 1998). The reverse may be true for secondaries that might accrete mass from their primaries. In any case, at least one of the stars in the binary must be massive in the above sense to qualify the binary to be "massive".

\subsection{Interacting vs. non-interacting binaries}

A crucial distinction among massive binaries is whether the two stars interact or not during their evolution. Starting at the ZAMS, where we assume the binary is non-interacting (otherwise it would not be stable and exist as such for very long), there will be no subsequent interaction affecting either star's evolution for initial periods greater than $P_{c} \sim 10$ years. The exact critical period will depend on various factors, like eccentricity, masses, spin, etc. On the other hand, colliding winds (CW) will occur even for very large separations or long periods far in excess of 10 years, where X-ray and other emission from the 
CW zone will go as $1 / D$, where $D$ is the orbital separation. Apart from CWs, the two stars in this case will behave almost as if each was single.

On the other hand, for $P<P_{c}$, not only will CWs become more intense (but not necessarily following a $1 / D$ law for very small $D$, where the winds could collide at sub-terminal speeds, and other complications arise, e.g. non-adiabatic processes, heating effects), but (normally) the primary star can either fill its Roche lobe and start spilling over to the secondary, or the primary can engulf the secondary in a Common Envelope phase. The former leads (for the most extreme conservative case) to an increase in the separation and the period, while the latter invariably leads to a dramatic decrease in separation, often to a merger.

\subsection{RLOF or $C E$ ?}

Whether RLOF or CE ensues after the ZAMS depends on the mass-loss rate of the primary while it is expanding at its Roche lobe. Generally speaking for massive binaries, if the rate exceeds $\sim 10^{-3} M_{\odot} y r^{-1}$, then $\mathrm{CE}$ will be favoured (Vanbeveren et al. 1998).

As long as a single massive star can reach the red-supergiant (RSG) phase, in a binary such a star will eventually carry out RLOF if $P<P_{c}$. This was believed to be the case for stars up to $\sim 40 M_{\odot}$; for stars above this limit, their LBV phase guarantees that they will not do RLOF. However, with the revision in the masses of RSGs in the Local Group down from 25-40 to 10-25 $M_{\odot}$ (Massey et al. this volume), implies that there will be no RLOF for most stars in binaries with primary masses above $\sim 25 M_{\odot}$, with the reasonable assumption that an LBV phase occurs instead of RSG for those initial masses. During the LBV phase, the average mass-loss rate is of order $10^{-4} M_{\odot} y r^{-1}$, depending probably mostly on the mass. However, what really counts is the episodic mass-loss rate, which can be orders of magnitude higher, when the star is also most puffed up. In those cases, huge quantities of mass can be lost from the system in such short-lived bloated stages, as witnessed by the (admittedly extreme) LBV binary $\eta$ Car $(\mathrm{P}=5.54$ yrs; Corcoran et al. 2007). Unfortunately, little is known about the details of such general behaviour in LBVs, especially concerning the frequency and intensity of their eruptions.

Luckily, though, there are some empirical tests of whether RLOF or CE has taken place. One test, recently completed, is the evaluation of the binary frequency among WR stars in different metallicity environments. WR stars are like the canary in the mine, quite sensitive to metallicity-driven winds leading up to their formation. As one goes from the Solar neighbourhood in the Galaxy $\left(Z_{\odot}\right)$ to the LMC $\left(Z_{\odot} / 2\right)$ and the SMC $\left(Z_{\odot} / 5\right)$, one expects, even allowing for increased rotation at low metallicity $(\mathrm{Z})$, that the lower limit for the formation of single WR stars will increase systematically (Maeder \& Meynet 2000). This means that, assuming a binary frequency that is independent of metallicity and assuming short-period binaries will remove the outer layers of massive stars down to some fixed mass limit independent of $Z$, one expects to find significantly increasing WR+O binary frequency as one goes from the Galaxy to the LMC and SMC. Schnurr et al. (2008) have just finished a comprehensive search for WR binaries among the WNL population of the LMC, completing the previous studies of Foellmi et al. $(2003 \mathrm{a}, \mathrm{b})$ for Magellanic Cloud (MC) WNE stars and Bartzakos et al. (2001) for MC WC stars. (There are no WNL stars in the SMC.) Using the very laborious but necessary technique of searching for periodic RV varitions as signatures of $\mathrm{WR}+\mathrm{O}$ binaries, we now have a complete, uniform study of all 144 WR stars in both MCs.

Table 1 summarizes the results for these stars, except for the 6 bright WNL stars in R136 (see below), compared to all the well-studied WR stars in the solar neighbourhood. This table shows no compelling trends of binary frequency with metallicity in any WR subgroup. (As an aside, it is curious to note that WN8 and WN9 stars appear to avoid 
Table 1. Wolf-Rayet binary frequency $(\mathrm{P}<200 \mathrm{~d})$.

\begin{tabular}{l|c|c|c}
\hline Group & Galaxy $^{1}\left(\mathbf{Z}_{\odot}\right)$ & $\mathbf{L M C}\left(\mathbf{Z}_{\odot} / \mathbf{2}\right)$ & $\mathbf{S M C}\left(\mathbf{Z}_{\odot} / \mathbf{5}\right)$ \\
\hline $\mathrm{WN}$ & $9 / 30=0.30$ & $16 / 102=0.15$ & $4 / 11=0.36$ \\
\hline $\mathrm{WC} / \mathrm{O} \mid$ & $7 / 38=0.18$ & $3 / 24=0.13$ & $(1 / 1=1.00)$ \\
\hline $\mathrm{WR}$ & $16 / 68=0.24$ & $19 / 126=0.15$ & $5 / 12=0.42$ \\
\hline $\mathrm{WN} 8,9$ & $0 / 4=0.00$ & $0 / 8=0.00$ & not present \\
\hline
\end{tabular}

Notes:

${ }^{1}$ for $\mathrm{d}<4 \mathrm{kpc}$ and $6.5<\mathrm{R}(\mathrm{kpc})<9.5$, assuming $\mathrm{R}_{o}=8 \mathrm{kpc}$.

binaries, but they do tend to be runaways, both suggesting a peculiar formation mechanism.) Although the metallicity only varies over a factor $\sim 5$ here, the Z-dependence for minimum masses to become single WR stars is significant. We conclude that RLOF is not a significant factor in the formation of WR stars, contrary to what has been believed in the past (Vanbeveren et al. 1998). This may appear strange, especially given the reduction in mass-loss rates of $\mathrm{O}$ stars, the immediate progenitors of WR stars, by a factor $\sim 3$ (just like for WR stars: Moffat 2008). So how do WR stars form? One possibility is that the crucial phase to bring an $\mathrm{O}$ star to become WR, binary or single, is the LBV phase, where enormous quantities of mass are lost, possibly via continuum driving independently of metallicity (Smith \& Owocki 2006).

Another prediction of RLOF is that after RLOF, all upper layers of the WR progenitor are removed, leaving zero $\mathrm{H}$ in the final WR star. Foellmi et al. (2006) have studied the eclipsing WN3(h)a + O5 LMC binary BAT99-129, with $\mathrm{P}=2.77 \mathrm{~d}$. The WR component here has $\mathrm{H} / \mathrm{He} \sim 1$ by number, making it highly unlikely that RLOF has occurred (however, see Langer \& Petrovic 2007).

We take all of this to mean that, although RLOF can occur in some very tight massive systems, it is not generally common in systems that lead to most WR binaries. CE may be much more common, explaining the existence of numerous short-period $\mathrm{WR}+\mathrm{O}$ systems. Of particular interest are the two $\mathrm{WC} 4+\mathrm{O}$ systems $\mathrm{Br} 31$ and $\mathrm{Br} 32$ in the LMC, with $\mathrm{P}=3.0 \mathrm{~d}$ and $1.9 \mathrm{~d}$, resp. (Moffat et al. 1990). RLOF is unlikely responsible for short periods like this in such highly evolved binaries; rather, CE evolution, probably during the LBV phase, is the most likely source.

\section{Statistics}

Still of considerable relevance is the O-star binary frequency study of Mason et al. (1998), who found an essentially constant binary frequency for short-period systems below $P \sim 1$ month and above $P \sim 10^{4}$ yrs. In between there is a wide, deep dip in numbers, where the usual techniques of spectroscopic RV-orbit and visual-binary determination are difficult. In the likely case that the gap will eventually be filled in, it thus appears very likely that Öpik's power-law also applies to O binaries for all separations, i.e. $f(s) d s \propto$ $s^{-1} d s=d \log _{e}(s)$, and the same flat law in log $\mathrm{P}$, since Kepler's 3rd law has powerlaw coupling between $P$ and $s$. Furthermore, the overall initial binary frequency of $\mathrm{O}$ stars is probably close to $100 \%$, at least in clusters where all $\mathrm{O}$ stars are believed to be formed, with the additional trend that short-period systems favour mass ratios close to order unity. Unfortunately, star-formation theory including binaries (e.g. Bonnell 2007) is unclear how to reproduce these binary trends for massive stars.

If we now adopt Öpik's law as being the same for all (high) masses, then we will have a binary frequency on the $\mathrm{MS} N(P)=k d(\log P)$, with $k=$ const, for periods lying in 
the range $P_{\min }<P<P_{\max }$. Then, assuming that all (massive) binaries with $P<P_{\mathrm{sp}}$ can be detected with current spectroscopic means, then the ratio of that number to the total will be $R=\log \left(P_{\mathrm{sp}} / P_{\min }\right) / \log \left(P_{\max } / P_{\min }\right)$. Thus, taking reasonable values $P_{\min } \sim$ $1 \mathrm{~d}, P_{\mathrm{sp}} \sim 1 \mathrm{yr}$ and $P_{\max } \sim 10^{7} \mathrm{yr}$ (corresponding to a separation of $\sim 1 \mathrm{pc}$ in a cluster where most massive stars are born), yields $\mathrm{R} \sim 0.3$. This ratio is much like the typical spectroscopic binary frequency of massive stars observed in young clusters (Sana et al. this volume) and is thus compatible with $\sim 100 \%$ binary frequency in the birth process of massive stars.

Although it will take some time, attempts are well underway to fill the Mason et al. gap (if real) for intermediate periods (e.g. Gamen et al. 2008; Maiz Appellaniz et al. 2008). Of particular interest is the overlap between spectroscopic and visual binaries (leading to precise distances and masses), which currently is essentially negligeable. However, there is one case that deserves special mention: the recent detection of a visual orbit for the closest known WR star, $\gamma^{2}$ Vel, that happens to be in a well-known, 78.53d-spectroscopic binary (North et al. 2007)-see Fig. 1. A revised and more precise distance of $336_{-7}^{+8}$ pc was obtained, compared to $258_{-31}^{+41}$ pc from Hipparcos (Schaerer et al. 1997, van der Hucht et al. 1997). The O-star radius $\mathrm{R}_{O}=17 \pm 2 R_{\odot}$ is now compatible with an O7.5II-III star, compared to O7.5III-V with Hipparcos. The masses are $28.5 \pm 1.1$ and $9.0 \pm 0.6 \mathrm{M}_{\odot}$ for the $\mathrm{O}$ and WC8 components, respectively. This is the first time ever that a complete visual orbit has been obtained for a WR+O binary, finally opening the door to obtaining truly reliable parameters to constrain WR-star models.

\section{Life without massive binaries}

Massive binaries are not just twice as interesting as single stars. Due to a whole host of interaction effects, some even at relatively large separations, massive binaries lead to a large variety of phenomena in the Universe that would simply be lacking if massive binaries did not exist. Inspired by a recent workshop devoted to Massive Stars in Interacting Binaries (MSIB, St-Louis \& Moffat 2007), here are ten grand ways that life in the Universe would be less interesting without massive binaries:

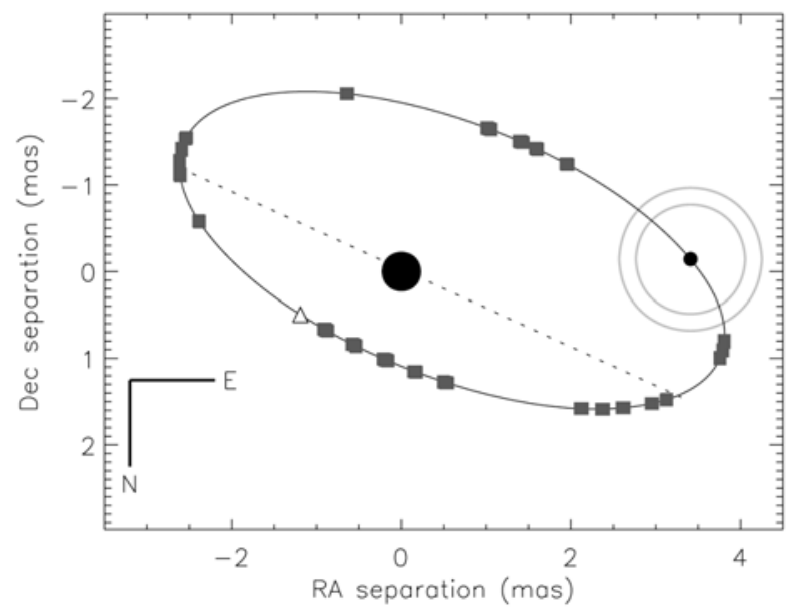

Figure 1. Visual orbit of $\gamma^{2}$ Vel relative to the O star (from North et al. 2007). 


\subsection{Enhanced stellar $X$-rays}

In very young stellar clusters, the most luminous X-ray sources are often massive binaries. A good example of this is the Carina Nebula (Corcoran et al. 1995), in which the dominating X-ray sources are $\eta$ Car, WR25, HD 93250, HD93205 and HD 93129, all relatively close binaries in which the X-ray flux enhancement is due to colliding winds $(\mathrm{CW})$. The clusters in the Carina Nebula are too young to have already made X-ray binaries with accreting compact companions. The mutual interaction of all the hot-star winds in young clusters also leads to a diffuse X-ray background, best seen in the very dense, 1 Myr cluster NGC 3603 (Moffat et al. 2002).

CW effects have been found to be especially important in three key systems. Nazé et al. (2007) determined the first X-ray light curve in an extragalactic binary system, HD 5980 ( LBV + WNE, P $=19.3 \mathrm{~d}$, e $=0.3$, with deep optical eclipses): the CW Xray flux reaches a maximum when the star with the faster, less-dense wind (the WNE companion) passes in front of the $1994 \mathrm{LBV}$-erupter, as expected. Corcoran et al. (priv. comm.) have compared the X-ray light curves of $\eta$ Car (LBV + WNE, $\mathrm{P}=5.54 \mathrm{yr}, \mathrm{e} \sim 0.9)$ and WR 140 (WC7pd + O5I, P = $7.94 \mathrm{yr}, \mathrm{e}=0.88)$ : both systems show a general $1 / D$ dependence (although asymmetric), broken by deep absorption due to eclipses when the WNE star (wide dip in $\eta$ Car) or the O5 star (narrow dip) passes in front of the primary star and eclipses most of the CW X-ray emitting zone. I call attention that both $\eta$ Car and WR 140 will be going through their very active periastron passages, lasting only a few months, centred on January 2009, the International Year of Astronomy! During and around that interval, various observing campaigns using different techniques are being organized.

In slightly older clusters, high-mass X-ray binaries (HMXRB) and X-ray emitting supernova remnants (SNR) begin to appear, as the more massive stars complete their evolution. In general, the most frequent type of HMXRB are those containing $\mathrm{Be}+\mathrm{NS}$ (Negueruela 2007). Some recent X-ray accretion highlights are the following:

- FUSE observations have revealed the Hatchett-McCray ionization effect for the first time in the HMXRB with the highest-mass OB star known, HD 153919, O6Iaf + NS or BH (associated with 4U1700-37) (Iping \& Sonneborn 2007).

- After the only reliably established WR + cc system so far, Cyg X-3 (P=4.8h) in the Galaxy, two other similar cases have now been discovered, each with orbital periods just over 30d: IC 10 X-1 (Bauer \& Brandt 2004) and NGC 300 X-1 (Carpano et al. 2007). Their rarity is reflected in the fact that one had to go well beyond the Galaxy to find other cases.

- A TeV gamma-ray light curve has been obtained for the first time for a HMXRB, that of the microquasar LS 5039, with a $3.9 \mathrm{~d}$ orbital modulation as seen at other more conventional energies (Aharonian et al. 2006)-see Fig. 2. Phase-dependent inverse-Compton scattering is believed to be the source.

\subsection{Non-thermal radio emission}

The fraction of non-thermal (NT) radio emitters among OB and WR stars is quite high, of order $\sim 30 \%$. Since single stars are believed to produce only thermal radio emission arising in the $\sim 100 \mathrm{~km} / \mathrm{s}$ shocks associated with turbulent clumping, those systems that have an additional NT component are believed to be binaries in which the NT emission arises in the strong CW shocks between the winds (at $\sim 1000 \mathrm{~km} / \mathrm{s}$ ) of the two stars (Dougherty \& Williams 2000). This has been borne out recently in many systems, both spatially resolved and unresolved, the latter by monitoring the radio flux and its spectrum around the orbit. Probably the most impressive result was obtained for WR140, whose multi-frequency radio emission has now been resolved using VLBA (Dougherty et al. 
2005). The time-dependent behaviour of the bean-shaped NT emission coming from the $\mathrm{CW}$ bow head at phases when the radio emission is not attenuated, has been combined with the optical orbit (Marchenko et al. 2003) to deduce the complete orbit of WR140.

\subsection{Dust spirals}

Starting with WR104, WC9d, P = 241.5d (Tuthill et al. 1999, Tuthill et al. 2008), many dust-spirals have now been resolved in the NIR, always around pop I WC stars, either of type WC9 (and a few WC8; possibly always binary?) or other hotter WC types (always binaries). Recently, two more dust spirals have been imaged in the Quintuplet cluster (Tuthill et al. 2006); possibly all of the 5 extremely red stars in this cluster (from which it derives its name) are in fact dust spirals, some better resolved than others. In all cases, it is believed that the amorphous carbon-dust is formed by successive nucleation processes downstream in the CW shock cone of some massive binaries containing a WC star, where conditions are conducive to its formation.

Of particular interest in the case of WR140 from the VLBA data (see above) is that at periastron passage, the $\mathrm{O}$ star is located NW of the WR component on the sky, in a direction at odds with where one sees the bulk of the CW-produced dust emission to occur $(\sim \mathrm{SE})$. This surprising result suggests that conditions around the stars may be too hot to produce significant dust around periastron passage; most of the dust is probably produced at phases intermediate between periastron and apastron (Williams et al., in prep.).

Spectacular images have now been secured in the MIR for several dust spirals by Marchenko \& Moffat (2007). Of special note is the system WR112, WC9d, surrounded by 5 distinct spirals, believed to be due to the dust formed and carried out with the general shock-cone flow, during successive orbits - see Fig. 3. However, both here and in other objects (e.g. WR140; WR48a, WC8ed, P >20 yrs), the spirals are broken up into several (4?) arcs, each homologously repeating faithfully as the dust spiral expansion proceeds. One mechanism to explain this is currently being studied by Hervé et al. (in prep.), involving axisymmetric winds (with stronger and faster winds at the poles: Owocki 2004) whose rotation axes are misaligned both with each other and with the orbital binary axis of revolution. This can in principal produce two pairs of directions of enhanced CW effects including dust production and emission, with arbitrary angle between the two pairs.

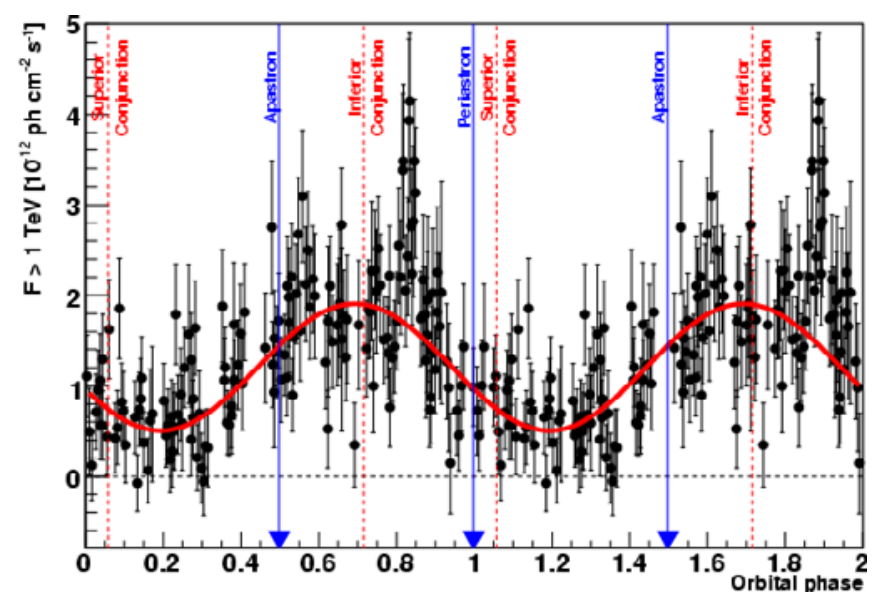

Figure 2. TeV light curve of microquasar LS 5039 (from Aharonian et al. 2006). 


\subsection{Inverse mass ratios}

For the 7 next grand ways of what we would miss without massive binaries, I will necessarily be brief, partly because it is beyond my own expertise, but also because these are discussed in more detail in the MSIB workshop (St-Louis \& Moffat 2007).

Besides the classic case of $\beta$ Lyr, where the mass-losing star in the interacting binary is the less massive, a more recent and equally interesting example is RY Scuti, which appears to be going through $\mathrm{CE}$ evolution on the way to become a close $\mathrm{WR}+\mathrm{O}$ binary (Smith et al. 2002; Grundstrom et al. 2007). Currently, the less massive star in RY Scuti is the more active mass-losing component.

\subsection{Very rapid spin}

Several types of stars may well owe their extreme spins to massive binary evolution: millisecond pulsars, Be stars and Gamma-Ray Bursts (see MSIB).

\subsection{Rejuvenation, blue stragglers}

The colour-magnitude diagrams (CMDs) of some young open clusters often reveal the presence of member stars that are located on younger isochrones to the blue side of the bulk of the cluster Main Sequence (e.g. Ahumada \& Lapasset 2007). However, in extremely young clusters, like NGC 3603 (age $1 \mathrm{Myr}$ ), the upper MS is essentially vertical (especially in observed colour indices), so one should talk not of Blue Stragglers, but rather "Luminous Stragglers", i.e. very massive stars that might have been rejuvenated to higher luminosities, without becoming significantly bluer, by binary merger or "cannibalism". Another important question is whether rejuvenated ("second phase") WR stars exist in as large numbers as claimed by Vanbeveren et al. (1998) via binary separation. Presumably, most of them become single runaways, that would populate the field between their preferred formation places in young star clusters. However, the runaway nature of field WR stars remains to be systematically demonstrated.

\subsection{Enhanced dense-cluster dynamics}

Massive stars are known to have large cross sections when it comes to stellar collisions in dense systems. This is even more the case when the massive stars occur in binaries.

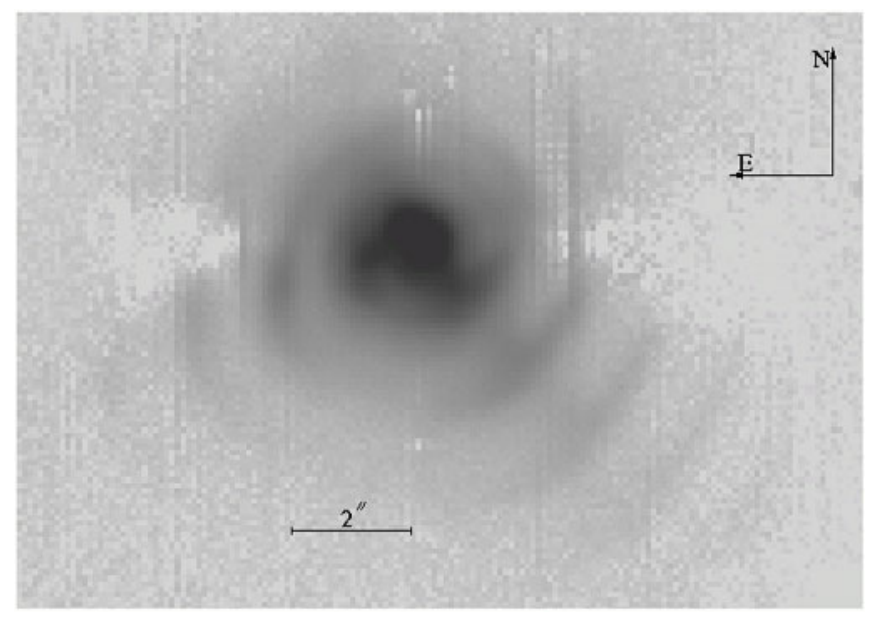

Figure 3. Mid-IR image of dust spirals around WC9d star WR112 (from Marchenko \& Moffat 2007). 
The accelerated cluster dynamics by massive binaries can have important consequences on the lifetimes and fates of such clusters (see MSIB).

\subsection{Runaways}

In contrast to lower-mass stars, O stars have a relatively high frequency $(\sim 25 \%)$ of being runaways, most likely leading to their presence in the field. Two independent processes probably operate to produce massive runaways, that are mostly single stars: SN in a close binary and the cluster "slingshot" effect (see MSIB). The fastest measured runaway WR star still appears to be WR124, with a peculiar radial velocity component of $\sim 200 \mathrm{~km} / \mathrm{s}$ (Moffat et al. 1982).

\subsection{IMBHs and possibly even $S M B H s$}

Fabbiano (2006) defines ultra-luminous X-ray sources (ULX) as those having $L_{X}>10^{39}$ $\operatorname{erg~s}^{-1}$. If this arises in a single source, then that object must be a black hole $(\mathrm{BH})$ with mass $>100 \mathrm{M}_{\odot}$, i.e. an intermediate-mass $\mathrm{BH}$, compared to stellar-mass BHs. Such IMBHs would bridge the gap between stellar (mass $<30 \mathrm{M}_{\odot}$ ) and supermassive BHs found in the centres of large galaxies, both in mass and in X-ray spectral hardness. Perhaps IMBHs are precursors to SMBHs. IMBHs could be either the collapsed remnant of a massive primordial star in the early Universe, or formed in the core-collapse of a young, dense star-cluster, ultimately facilitated by massive-binary effects. If these ULXs are not IMBHs, then they could represent the upper limit of a normal XRB population.

\subsection{Gamma Ray Bursts}

While the best candidates for the short, hard GRBs are merging $\mathrm{NS}+\mathrm{NS}$ or $\mathrm{NS}+\mathrm{BH}$ (not $\mathrm{BH}+\mathrm{BH}$, however) binaries, the more intense, long, soft bursts appear to involve the collapse via SN Ic of a rapidly spinning WR progenitor (core) most likely of type WC/WO. The short, hard GRBs involve massive-binary progenitors, while the long, soft GRBs might also, in order to sufficiently spin up the collapsing star. WC/O + O binaries with initial periods $P<3$-4d might meet the bill, given that no single WR star is known currently to be spinning fast (van den Heuvel 2007). As noted in Section 1, such binaries do exist, e.g. Br 31 and $\mathrm{Br} 32$ in the LMC are WC4 + O6 systems with periods of 3.0d and 1.9d (Moffat et al. 1990). Recent evidence of the alternative scenario of single WC/WO stars that remain spinning fast at low metallicity shows that this bias cannot be strong, if it exists at all (Fynbo et al. 2006).

\section{Physical parameters of stars}

Binaries are excellent tools for determining various stellar parameters because they act as yardsticks. I will concentrate here on the recent exciting example of the quest for the masses of the most massive stars in the local Universe. This is important for the following reasons:

- to fix the L(M) relation, which should flatten out at high M;

- to learn about the physics of MS stars near their Eddington limit;

- to provide links to population III stars, expected to be on average at least an order of magnitude more massive than today's average;

- to help decide whether the upper mass-limit for stars is due to the star-formation process or petering out of the IMF.

The bottom line appears now to be that the most massive stars in the Local Universe are of type WN5-7ha. These are not classical He-burning WR stars; they are rather often found to be the most luminous H-burning MS stars known. Luckily, such stars, although 
extremely rare, are intrinsically very luminous, allowing them to be found in a larger volume. Because of this and the huge winds they produce, their orbital inclinations can be obtained when in binaries, via four different time-dependent techniques: (1) (normally double per orbit) photospheric eclipses, (2) single-dip atmospheric (continuum) eclipses (Lamontagne et al. 1996), (3) broadband linear polarization (St-Louis et al. 1988), and (4) wind-wind collisions (Hill et al. 2002). This has advantages when it comes to determining stellar masses.

Where does one find such stars? According to some star-formation scenarios, the most massive stars tend to lie near the dense cores of rich clusters. However, the true situation may be more complicated, since one does find some of the earliest (thus massive) stars in much looser, young associations and outside the central dense parts of clusters/associations. A good example of this are the two bright WN6h stars in 30 Dor, R144 (believed to be the brightest WR star in the Local Group: Crowther, priv. comm.) and R145 (see below), that are located well away from the central R136 core. Another example is the rich but sparse association Cyg OB2, which contains some $150 \mathrm{OB}$ and WR stars. It is also a good idea to look among the brightest MS stars of the youngest dense clusters, since evolved stars have their own complications.

\subsection{NGC 3603/A1, C}

The best example of a dense, young, rich cluster in the Galaxy is NGC 3603, with age 1 Myr (Brandl et al. 1999; Melena et al. 2008) and the bulk of its stars within a radius of $\sim 30 "$ (1 pc for a distance of $7 \mathrm{kpc}$ ). Its HST-based RI CMD (Drissen 1999) reveals a tight upper MS, with 3 WN6ha stars (A1, B, C) located on average a full magnitude above the brightest cluster $\mathrm{O}$ stars, reaching the earliest known types of O2-3. Since these H-rich $\left(X_{H} \sim 0.6\right.$ : Crowther \& Dessart 1998) WNL stars are now believed to have similar $T_{\text {eff }}$ compared to O2-3 stars (Crowther, priv. comm.), this makes the WNL stars in NGC 3603 very luminous and hence potentially very massive. They also lie within 2" $(0.06 \mathrm{pc})$ of the cluster centre.

Schnurr et al. (in prep.) have now obtained repeated (22 visits over 8 months during 2005) K-band spectra of the 3 WN6ha stars and two central bright O3 stars in NGC 3603, using IFU + AO in SINFONI at the VLT. Without such high spatial resolution, this would have been impossible in the tight core of NGC 3603. Schnurr et al.'s spectra confirm the binary nature of star A1 (with two unequally bright WN stars), for which deep double eclipses in a $\mathrm{P}=3.7724 \mathrm{~d}$ orbit were found previously from HST/NICMOS photometry (Moffat et al. 2004), leading to an orbital inclination $i=71^{\circ}$. Combined with the RV orbits, this yields masses of $114 \pm 30$ and $84 \pm 15 \mathrm{M}_{\odot}$ for A1. (Further observations are underway with the hope to improve on this estimate.) Then, while star B was found to have constant RV over a year, star C appears to be a short-period (9d) single-line spectroscopic binary with significant eccentricity $(\mathrm{e}=0.3)$. With $L_{X}>4 \times 10^{34} \mathrm{erg} \mathrm{s}^{-1}$, star $\mathrm{C}$ has the highest known X-ray flux of any Galactic WR star, apart from the extremely eccentric-orbit system WR140 at X-ray maximum. Even A1 has $L_{X}>10^{34} \mathrm{erg} \mathrm{s}^{-1}$, while star B only has $L_{X}=10^{33} \mathrm{erg} \mathrm{s}^{-1}$. All of this is compatible with $\mathrm{CW}$-enhanced X-rays, although it may appear surprising that WR binaries of such short period yield such high X-ray fluxes. The reason for this is probably that A1 and C contain MS WNLh stars, quite different from the more compact, classical dense-wind (although not necessarily greater mass-loss rate) He-burning WR stars.

\subsection{Westerlund 2/WR20a}

Another luminous star that has turned out to have high masses is WR20a, with identical, eclipsing components WN6ha + WN6ha in a $\mathrm{P}=3.675$ d circular orbit (Rauw et al. 2004, 
2005; Bonanos et al. 2004). It is a member of a moderately dense, young cluster, also containing early-O type stars, Westerlund 2, although WR20a is not located near its core. The masses are $83 \pm 6$ and $82 \pm 6 \mathrm{M}_{\odot}$, which before NGC 3603/A1, made them the most massive stars ever "weighed" in a binary. The position on the HRD of these two stars also fits well the isochrones for $77 \pm 4 \mathrm{M}_{\odot}$, although the models used for comparison do not include rotation.

\subsection{Carina Nebula/WR25}

The Carina Nebula and its several young clusters of varying ages also contains three WNLh stars (WR22, 24, 25), much like those in NGC 3603. However, none of these 3 stars is located at or near the core of any of the Carina Nebula's clusters. On the other hand, as in NGC 3603, two of the Carina Nebula's WNLh stars are binary, although of much longer period, while the third appears to be single (WR24). While WR22's WNLh component is of "only" moderately high mass $\left(55 \mathrm{M}_{\odot}\right.$ according to Schweickhardt et al. 1999; $72 \mathrm{M}_{\odot}$ from Rauw et al. 1996; $\left.\mathrm{P}=80.3 \mathrm{~d}\right)$, WR25 now finally has a long-awaited orbital solution (Gamen et al. 2006; Gamen et al. 2008): $\mathrm{P}=207.7 \mathrm{~d}$, e $=0.56, \mathrm{M} \sin ^{3} \mathrm{i}$ $=75 \pm 7,27 \pm 3 \mathrm{M}_{\odot}$. However, the only source of orbital inclination so far is from WR25's X-ray light curve (Pollock 2008), with $i=37 \pm 2^{\circ}$, which yields implausibly high absolute masses. For comparison, Hamann et al.'s (2006) atmospheric models for WR25 yield $L=10^{6.8} \mathrm{~L}_{\odot}, T_{\star}=50 k K$, making it much closer to $\eta$ Car in the HRD. The modeled mass is either 110 or $210 \mathrm{M}_{\odot}$, depending on the adopted distance $\left[(\mathrm{m}-\mathrm{M})_{o}=11.8\right.$ (à la Walborn) or 12.55 (à la Massey)].

\subsection{Dor/outside R136}

In his detailed spectroscopic search for, and study of, all the WNL binary stars in the LMC, Schnurr (2007) (see also Schnurr et al., in prep.) finds that two stars stand out as being especially luminous and thus potentially very massive, both in the 30 Dor region (although not in the central dense cluster R136): R144 and R145. Both are WN6h but, as noted above, R144 is considered to be the most luminous WR star in the Local Group. With a likely long orbital period, it is still under study. R145 on the other hand, is about 0.5 mag fainter than R144 in intrinsic brightness, but now possesses a plausible $\mathrm{RV}$ orbit, with $\mathrm{M} \sin ^{3} \mathrm{i}=140 \pm 37,59 \pm 26 \mathrm{M}_{\odot}$ for its two components. However, with $i=40 \pm 6^{\circ}$ from broadband linear polarimetry, one again obtains implausibly high masses. Furthermore, this system shows strong phase-dependent variation in CW excess emission in its HeII 4686 emission line, which appears to be compatible with this lowinclination value. The source of the problem of high masses (e.g. in the shift-and-add technique to extract the RV orbit of the faint companion?) is under investigation.

\subsection{Dor $/ R 136$}

Schnurr et al. (in prep.) have also monitored for RV variations all of the 6 most luminous (all WNL-like) stars within R136 (i.e. R136a1, a2, a3, a5, b, c). Based on 9 spectra for each object spread over 22 days using VLT/SINFONI in NIR/AO spectroscopic mode, they find that none of these stars shows RV variations above the $\sigma \sim 20 \mathrm{~km} / \mathrm{s}$ level (worse for a5, which has weak lines). This is in contrast with the expectation of Moffat et al. (1985) that one component in R136 revealed (heavily diluted) binary motion with $\mathrm{P}=4.377 \mathrm{~d}$. This result, although subject to small numbers, is in stark contrast with the central WNL stars in NGC 3603, where $2 / 3$ are short-period binaries, and the Carina Nebula, where $2 / 3$ are moderately long-period binaries. In addition, none of these 6 stars in R136 shows any evidence for runaway motion. 
Table 2. Summary of luminous WN5-7h(a) stars $\left(\mathrm{L}>\mathrm{L}_{\odot}\right)$ with Keplerian mass estimates.

\begin{tabular}{|c|c|c|c|c|c|c|c|}
\hline Star & Spectrum & $P(d)$ & e & $\mathrm{i}\left({ }^{o}\right)$ & $\mathrm{M} \sin ^{3} \mathrm{i}\left(\mathrm{M}_{\odot}\right)$ & $\mathrm{M}\left(\mathrm{M}_{\odot}\right)$ & $\mathrm{L}_{X}\left(\operatorname{ergs}^{-1}\right)$ \\
\hline $\begin{array}{l}\text { Carina Nebula } \\
\text { WR } 22 \\
\text { WR } 25\end{array}$ & $\begin{array}{c}\text { WN7h+O9V-III } \\
\text { WN } 6 \text { ha+O }\end{array}$ & $\begin{array}{c}80.3 \\
207.7\end{array}$ & $\begin{array}{l}0.60 \\
0.56\end{array}$ & $\begin{array}{l}90 \\
37\end{array}$ & $\begin{array}{l}55 / 21 \\
75 / 27\end{array}$ & $\begin{array}{c}55 / 21 \\
(344 / 124)\end{array}$ & $\begin{array}{ll}3 & 10^{32} \\
8 & 10^{33}\end{array}$ \\
\hline $\begin{array}{l}\text { NGC } \mathbf{3 6 0 3} \\
\text { A1 } \\
\text { C }\end{array}$ & $\begin{array}{c}\text { WN6ha+ WN6: } \\
\text { WN6ha+? }\end{array}$ & $\begin{array}{l}3.8 \\
8.9\end{array}$ & $\begin{array}{c}0 \\
0.3\end{array}$ & $\begin{array}{c}71 \\
?\end{array}$ & $\begin{array}{c}96 / 71 \\
?\end{array}$ & $\begin{array}{c}114 / 84 \\
?\end{array}$ & $\begin{array}{l}>110^{34} \\
>410^{34}\end{array}$ \\
\hline $\begin{array}{l}\text { Westerlund } 2 \\
\text { WR20a }\end{array}$ & WN6ha+ WN6ha & 3.7 & 0 & 74 & $74 / 73$ & $83 / 82$ & $810^{33}$ \\
\hline $\begin{array}{l}\text { 30 Dor } \\
\text { R144 } \\
\text { R145 }\end{array}$ & $\begin{array}{c}\text { WN6h } \\
\text { WN6h+O }\end{array}$ & $\begin{array}{c}\text { ?(long)? } \\
158.8\end{array}$ & $\begin{array}{c}? \\
0.68\end{array}$ & $\begin{array}{c}? \\
40\end{array}$ & $\begin{array}{c}? \\
140 / 59\end{array}$ & $\begin{array}{c}? \\
(519 / 218)\end{array}$ & $\begin{array}{ll}2 & 10^{33} \\
1 & 10^{33}\end{array}$ \\
\hline
\end{tabular}

Notes:

Masses are for the primary/secondary, resp.

\subsection{Summary}

Table 2 shows a summary of the 7 most likely candidates for the highest mass among LG stars. All of them are of type WN5-7h or WN5-7ha. Among O stars, none has been found to exceed $\sim 60 \mathrm{M}_{\odot}$ based on Keplerian orbits (Williams et al. 2008). Even the most luminous known O star, HD 93129A, O2If*, a visual binary, has masses estimated to be $\sim 80$ and $50 \mathrm{M}_{\odot}$ (Nelan et al. 2004). These WN5-7h/ha stars are not "classical" He-burning WR stars; rather they are a kind of super-Of star, whose optical emission lines are due to the extremely high stellar luminosity. Some are very young $(\sim 1 \mathrm{Myr})$, so they appear to represent an extension of the upper MS above that occupied by the hottest known O stars of types O2-3.

Why were these stars overlooked before as being the most massive? A likely reason is that with improved atmosphere models including blanketing effects, the hottest MS O stars have become $\sim 10 \%$ cooler and thus fainter, while WNLh stars have become hotter and brighter (Crowther, priv. comm.)-see Fig. 4 . Both seem to converge to $T_{\text {eff }} \sim 45 \mathrm{kK}$.

None of these stars gives a convincing Keplerian-based mass estimate that surpasses $\sim$

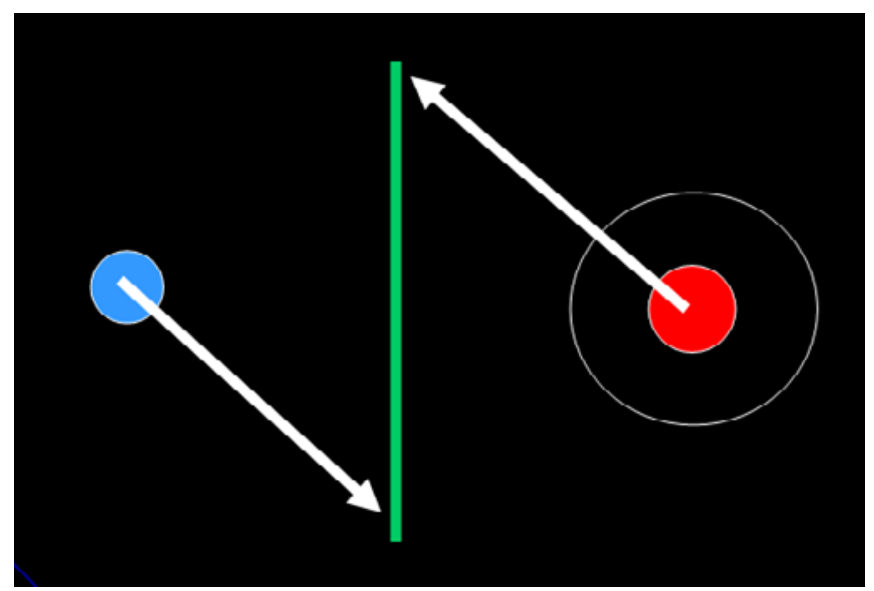

Figure 4. Schematic illustration in a CMD how models of luminous WNLh stars (right) have become hotter and brighter, while early-O stars (left) have become cooler and fainter, both converging to $\mathrm{T}_{\text {eff }} \sim 45 \mathrm{kK}$. 
$150 \mathrm{M}_{\odot}$. This is entirely compatible with other independent estimates of the upper limit of star formation in the local Universe, based on number statistics of luminous stars in massive, young clusters (Figer 2005; Weidner \& Kroupa 2004; Oey \& Clark 2005; Koen 2006).

\section{Final remark}

As in most areas of Astronomy these days, the study of massive stars is currently enjoying a particularly active and exciting epoch of discovery and relevance to the Cosmos as a whole. This is especially true for massive binaries!

\section{References}

Aharonian, F., Akhperjanian, A. G., Bazer-Bachi, A. R., et al. 2006, A\&A, 460, 743

Ahumada, J. \& Lapasset, E. 2007, A\& $A, 463,789$

Bartzakos, P., Moffat, A. F. J., \& Niemela, V. S. 2001, MNRAS, 324, 18

Bauer, F. E. \& Brandt, W. M. 2004, ApJ, 601, 67

Bonanos, A. Z., Stanek, K. Z., Udalski, A., et al. 2004, ApJ, 611, L33

Bonnell, I. A. 2007, St-Louis, N. \& Moffat, A. F. J. (eds.), Massive Stars in Interacting Binaries, (San Francisco: ASP) ASP Conf Series, 367, 303

Brandl, B., Brandner, W., Eisenhauer, F., et al. 1999, A\&SA, 352, 69

Carpano, S., Pollock, A. M. T., Wilms, J., et al. 2007, A\&A, 461, L9

Corcoran, M. F., Swank, J., Rawley, G., et al. 1995, Rev. Mex. A\&GA Conf. ser., 2, 97

Corcoran, M. F., Hamaguchi, K., Gull, et al. 2007, St-Louis, N. \& Moffat, A. F.J. (eds.), Massive Stars in Interacting Binaries, (San Francisco: ASP) ASP Conf Series, 367, 249

Crowther, P. A. \& Dessart, L. 1998, MNRAS, 296, 622

Dougherty, S. M. \& Williams, P. M. 2000, MNRAS, 319, 1005

Dougherty, S. M., Beasley, A. J., Claussen, M. J., et al. 2005, ApJ, 623, 447

Drissen, L. 1999, in: K. A. van der Hucht, G. Koenigsberger \& P. R. J. Eenens (eds.), WolfRayet Phenomena in Massive Stars and Starburst Galaxies, (San Francisco: ASP), Proc. IAU Symp, 193, 741

Dufour, P., Liebert, J., Fontaine, G., \& Behara, N. 2007, Nature, 450, 522

Fabbiano, G. 2006, ARA\&A, 44, 323

Figer, D. F. 2005, Nature, 434, 192

Foellmi, C., Moffat, A. F. J., \& Guerrero, M. A. 2003a, MNRAS, 338, 360

Foellmi, C., Moffat, A. F. J., \& Guerrero, M. A. 2003b, MNRAS, 338, 1025

Foellmi, C., Moffat, A. F. J., \& Marchenko, S. V. 2006, A\&A, 447, 667

Fynbo, J. P. U., Starling, R. L. C., Ledoux, C., et al. 2006, A\&\&A, 451, L47

Gamen, R., Gosset, E., Morrell, N., et al. 2006, A\&A, 460, 777

Gamen, R., et al. 2008, in: P. Benaglia, G. Bosch \& C. E. Cappa (eds.), Massive Stars: Fundamental Parameters and Circumstellar Interactions, Rev. Mex. A\& $A$ Conf. ser., in press

Grundstrom, E. D., Gies, D. R., Hillwig, T. C., et al. 2007, ApJ, 667, 505

Hamann, W.-R., Gräfener, G., \& Liermann, A. 2006, A\& A, 457, 1015

Hill, G. M., Moffat, A. F. J., \& St-Louis, N. 2002, MNRAS, 335, 1069

Iping, R. C., \& Sonneborn, G. 2007, St-Louis, N., \& Moffat, A. F. J. (eds.), Massive Stars in Interacting Binaries, (San Francisco: ASP) ASP Conf Series, 367, 459

Koen, C. 2006, MNRAS, 365, 590

Lamontagne, R., Moffat, A. F. J., Drissen, L., et al. 1996, AJ, 112, 2227

Langer, N., \& Petrovic, J. 2007, St-Louis, N. \& Moffat, A. F. J. (eds.), Massive Stars in Interacting Binaries, (San Francisco: ASP) ASP Conf Series, 367, 359

Maiz Apellaniz, J., Walborn, N. R., Morrell, N. I., et al. 2008, in: P. Benaglia, G. Bosch \& C. E. Cappa (eds.), Massive Stars: Fundamental Parameters and Circumstellar Interactions, Rev. Mex. A\&A Conf. ser., in press

Maeder, A. \& Meynet, G. 2000, ARA\&A, 287, 803 
Marchenko, S. V., Moffat, A. F. J., Ballereau, D., et al. 2003, ApJ, 596, 1295

Marchenko, S. V. \& Moffat, A. F. J. 2007, St-Louis, N. \& Moffat, A. F. J. (eds.), Massive Stars in Interacting Binaries, (San Francisco: ASP) ASP Conf Series, 367, 213

Mason, B. D., Gies, D. R., Hartkopf, W. I., et al. 1998, AJ, 115, 821

Melena, N. W., Massey, P., Morrell, N. I., \& Zangari, A. M. 2008, AJ, 135, 878

Moffat, A. F. J., Lamontagne, R., \& Seggewiss, W. 1982, A\&A, 114, 135

Moffat, A. F. J., Seggewiss, W., \& Shara, M. M. 1985, ApJ, 295, 109

Moffat, A. F. J., Niemela, V. S., \& Marraco, H. G. 1990, ApJ, 348, 232

Moffat, A. F. J., Corcoran, M. F., Stevens, I. R., et al. 2002, ApJ, 573, 191

Moffat, A. F. J., Poitras, V., Marchenko, S. V., et al. 2004, AJ, 128, 2854

Moffat, A. F. J. 2008, in: Hamann, W.-R., Feldmeier, A., \& Oskinova, L. M. (eds.), International Workshop on Clumping in Hot-Star Winds, (Potsdam: Universitätsverlag), in press

Nazé, Y., Corcoran, M. F., Koenigsberger, G., \& Moffat, A. F. J. 2007, ApJ, 658, 25

Negueruela, I. 2007, St-Louis, N. \& Moffat, A. F. J. (eds.), Massive Stars in Interacting Binaries, (San Francisco: ASP) ASP Conf Series, 367, 459

Nelan, E. P., Walborn, N. R., Wallace, D. J., et al. 2004, AJ, 128, 323

North, J. R., Tuthill, P. G., Tango, W. J., \& Davis, J. 2007, MNRAS, 377, 415

Oey, M. S., \& Clarke, C. J. 2005, ApJ, 620, 43

Owocki, S. P. 2004, in M. Heydari-Malayeri, P. Stee, \& J.-P. Zahn (eds.), Evolution of Massive Stars, Mass Loss and Winds, EAS Publ. Ser., 13, 163

Pollock, A. M. T. 2008, in: P. Benaglia, G. Bosch \& C. E. Cappa (eds.), Massive Stars: Fundamental Parameters and Circumstellar Interactions, Rev. Mex. A\&A Conf. ser., in press

Rauw, G., Vreux, J.-M., Gosset, E., et al. 1996, A\&A, 306, 771

Rauw, G., De Becker, M., Nazé, Y., et al. 2004 A $\& A, 420$, L9

Rauw, G., Crowther, P. A., De Becker, M., et al. 2005, A\& A, 432, 985

Schaerer, D., Schmutz, W., \& Grenon, M. 1997, ApJ, 484, L153

Schnurr, O. 2007, Ph. D. thesis, Univ. de Montréal

Schnurr, O., Moffat, A. F. J., St-Louis, N., et al. 2008, MNRAS, submitted

Schweickhardt, J., Schmutz, W., Stahl, O., et al. 1999, A\&A, 347, 127

Smith, N., Gehrz, R. D., Stahl, O., et al. 2002, ApJ, 578, 464

Smith, N., \& Owocki, S. P. 2006, ApJ, 645, 45

St-Louis, N., Moffat, A. F. J., Drissen, L., et al. 1988, ApJ, 330, 286

St-Louis, N. \& Moffat, A. F. J. 2007 (eds.), Massive Stars in Interacting Binaries, ASP Conf. Ser. 367 (MSIB)

Tuthill, P. G., Monnier, J. D., \& Danchi, W. C. 1999, Nature, 398, 487

Tuthill, P. G., Monnier, J. D., Tanner, A., et al. 2006, Science, 313, 935

Tuthill, P. G., Monnier, J. D., Lawrance, N., et al. 2008, ApJ, 675, 698

Vanbeveren, D., Van Rensbergen, W., \& De Loore, C. 1998, The Brightest Binaries, (Dordrecht: Kluwer Academic Publications)

van den Heuvel, E. P. J. 2007, St-Louis, N. \& Moffat, A. F. J. (eds.), Massive Stars in Interacting Binaries, (San Francisco: ASP) ASP Conf Series, 367, 549

van der Hucht, K. A., Schrijver, H., Stenholm, B., et al. 1997, New Astron., 2, 245

Weidner, C., \& Kroupa, P. 2004, MNRAS, 348, 187

Williams, S. J., Gies, D. R., Henry, T. J., et al. 2008, ApJ, submitted arXiv:0802.4232

\section{Discussion}

ZINNECKER: As you have shown, the most massive binaries are not found in the centres of dense, young clusters, but outside. I wonder why? Both dynamical and primordial mass-segregation would suggest otherwise. Do you have any thoughts on this puzzling, counter-intuitive result?

Moffat: Well, some most massive stars are in cluster cores (e.g. A1 in NGC 3603). But yes, one has R145 in 30 Dor well outside the R136 core. Then there is the loose, but 
O-star rich association Cyg OB2, where one finds very massive stars all over the place, albeit not WNLha.

Crowther: If R145 (WN6h + OB) has a minimum mass of $140 \mathrm{M}_{\odot}$, what would the expected mass of the much brighter R144 (WN6h) star be?

Moffat: R144 is $\sim 0.5 \mathrm{mag}$ in $M_{v}$ (or in $M_{\mathrm{bol}}$, since the intrinsic colours are similar) brighter than R145, so R144 should be even more massive, indeed. The difference could be reduced, however, if R144s components are more nearly of equal brightness (like those in WR 20a) than those in R145.

Maíz-Appellániz: The Hipparcos distance to $\gamma^{2}$ Vel actually agrees with the recent VLTI results. First, the Lutz-Kelker correction has to be applied. Second, the recent reduction of the Hipparcos data by Floor van Leewen has pushed it farther away.

Moffat: Thanks, that's great to hear!

RAUw: Concerning these very high masses that make some sense in terms of an upper mass limit, there is also the core of HD 15558 (De Becker et al. 2006, A\&A 456, 1121), where we find $M \sin ^{3} i$ well above $100 \mathrm{M}_{\odot}$. The primary star is O5 III and the period is 442 days. It seems quite strange that these systems with very large $M \sin ^{3} i$ are often found to have long orbital periods. This might indicate that there is something wrong in our interpretation of the radial velocities $(\mathrm{RVs})$ of these stars.

Moffat: Yes, this is unusual for an O5 III star. But long-period systems should be easier and more reliable in RV. That is, as long as one is able to piece together lots of necessarily shorter snapshot observing runs, where there may also be systematic effects occurring between different runs. For R144 and R145 we are working to rectify this using more contiguous observing over long time scales at one (small) telescope.

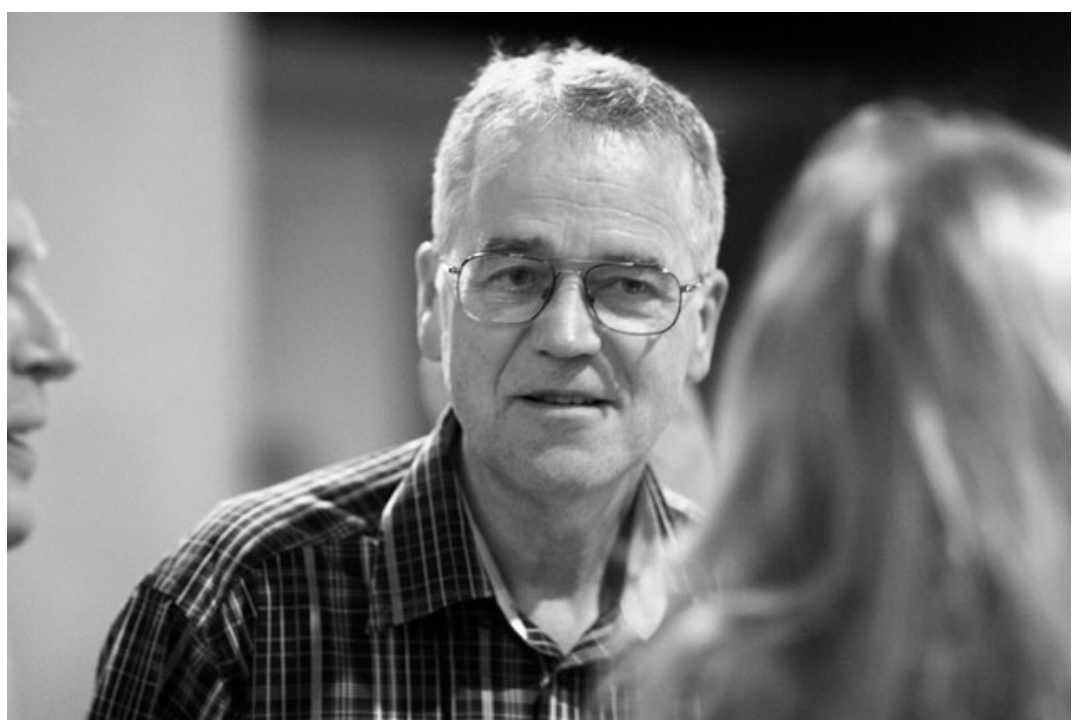

Tony Moffat. 